\title{
Multi-carrier Link-Layer Energy Efficiency and Effective Capacity Tradeoff
}

\author{
Wenjuan Yu, Leila Musavian and Qiang Ni \\ School of Computing and Communications, InfoLab21, \\ Lancaster University, Lancaster, UK, \\ Email: \{w.yu1, 1.musavian, q.ni\}@lancaster.ac.uk
}

\begin{abstract}
A joint energy and spectral efficient power allocation strategy for a point-to-point multi-carrier link, subject to a delayoutage probability constraint, is proposed in this paper. Since the two objectives, namely, link-layer energy efficiency (EE) and effective capacity (EC), conflict with each other, the tradeoff problem falls into the scope of multi-objective optimization problem (MOP). With the advanced lexicographic method, the MOP is converted into a single-objective optimization problem (SOP) by maximizing the multi-carrier EC, subject to an EE constraint. Then, by introducing an adjustable performance indicator, EE-loss-rate $\left(\alpha_{\mathrm{EE}}\right)$, into the $\mathrm{EE}$ constraint limit, the tradeoff level is flexibly controlled. Finally, we prove that the tradeoff formulation is a concave maximization problem and the optimum power allocation strategy is found using Lagrangian method. Analytical results indicate that the proposed power allocation has a similar structure to the one for EE-maximization problem over a frequency-selective fading channel, but with a different cut-off threshold.
\end{abstract}

\section{INTRODUCTION}

Green communication networks has received more and more attentions in recent years. The three major drivers are: a) increases in carbon dioxide emissions, b) increases in operational expenditures, and c) wider gap between the demand for energy and the offered battery capacity [1]. Firstly, the information and communication technology (ICT) sector is identified to be responsible for approximately 0.75 million tons of $\mathrm{CO}_{2}$ emissions for every 1 TeraWatt hour (TWh) energy consumption [2]. At the same time, it is known that ICT industry has the potential to reduce more than $23 \%$ of its current global green house gases (GHG) emissions [3]. Secondly, GHG effects cause lots of economical issues. In particular, if no action is taken, the overall costs and risks of climate change will be equivalent to losing at least $5 \%$ of global gross domestic product (GDP) every year [3]. In contrast, it is claimed that if one-third of the GHG emissions could be reduced, the generated economical benefit will be higher than the required investment [4]. Thirdly, the demand for wireless traffic is growing, while the improvement in battery technology is sluggish, about $10 \%$ increase every two years [5], as opposed to the power consumption of the processor, increasing by $150 \%$ every two years. This leads to a rapidly increasing gap between the demand for energy and the offered battery capacity. All these facts indicate that green communication networks are imperative.

Energy-efficiency (EE) metric, in $\mathrm{b} / \mathrm{J} / \mathrm{Hz}$, and spectral efficiency $(\mathrm{SE})$, in $\mathrm{b} / \mathrm{s} / \mathrm{Hz}$, have been considered as two key performance indicators for green wireless communication systems.

This work was supported in part by the China Scholarship Council, UK EPSRC under grant number EP/K011693/1 and the EU FP7 under grant number PIRSES-GA-2013-610524.
However, considering the compromise between the network performance and energy savings, how to balance the two conflicting objectives is a real challenge. The EE-SE tradeoff problem in a downlink orthogonal frequency division multiple access (OFDMA) network was considered in [6], which proposed an EE-maximization problem under a minimum overall throughput requirement and a series of minimum rate requirements. A joint power and subcarrier allocation scheme to maximize $\mathrm{EE}$ under minimum rate requirements for the service requested by each user, in cooperative cognitive radio networks, was proposed in [7]. A new green performance metric, namely, resource efficiency for EE-SE tradeoff, in a single cell downlink OFDMA network was introduced in [8], which used a weighted sum method to achieve an adjustable tradeoff formulation. For orthogonal frequency division multiplexing (OFDM) network with imperfect channel estimation, an adaptive power loading solution was provided in [9], which addressed the EE-SE tradeoff problem with changeable priority levels of EE and SE.

Overall, in the aforementioned studies, the system throughput was given by Shannon limit, which ignores the delay quality-of-service (QoS) requirements. However, for most mobile multimedia applications, which are delay-sensitive and power-consuming, such as video conferencing and online gaming, delay QoS requirement is a critical factor [10]. In this matter, link-layer effective capacity (EC) metric has been recently proposed to specify the maximum constant arrival rate under a delay-outage probability constraint [10]. Then, the link-layer EE could be defined as the ratio of EC to the total expenditure power. However, it is known that EE and EC-maximization approaches could be conflicting [10], [11]. Therefore, the compromise between the two metrics deserves elaborate study.

In this trend, using a curve fitting method, signal-to-noise ratio (SNR) was expressed in terms of EC in [12]. Then, $\mathrm{EE}$, as a function of SNR, and in turn, as a function of EC, was analyzed. Finally, EE-EC curve was provided in [12] and the impact of circuit power and QoS requirments on EEEC curve has been demonstrated by simulation results. An optimal power allocation strategy to maximize EC subject to a minimum EE constraint, for delay-limited mobile multimedia application was obtained in [13]. For the EE-EC tradeoff problems studied in [12] and [13], a frequency flat-fading channel was considered. However, these studies cannot be extended to multi-carrier systems. This is because despite the fact that the concavity and monotonicity of Shannon limit remains homogeneous for single-carrier and multi-carrier systems [6]-[9], EC of a multi-carrier system is not a linear summation of single-carrier's ECs. Henceforth, the concavity 
and monotonicity of EC, and the power allocation strategy of a single-carrier system could not be easily transplanted into a multi-carrier system.

This paper addresses the EE-EC tradeoff problem for a multi-carrier link over a frequency-selective fading channel, with a delay-outage probability constraint. Firstly, in order to jointly maximize $\mathrm{EE}$ and $\mathrm{EC}$, an MOP is formulated. Then, with the advanced lexicographic method, the MOP is solved by maximizing EC subject to an EE constraint. In order to flexibly control the constraint limit, and consequently control the tradeoff level, an adjustable indicator, referred to as EE-loss-rate $\left(\alpha_{\mathrm{EE}}\right)$, is introduced. The EE constraint is then defined as a product of $\alpha_{\mathrm{EE}}$ and the maximum achievable EE of the channel. We note that unlike Shannon capacity, the independent power allocation for each subcarrier will not provide an optimal solution for multi-carrier system. Therefore, a joint optimization over both frequency and time is necessary. Since EC function in multi-carrier systems is concave in the transmission power and the link-layer EE is quasiconcave, the proposed EE-EC tradeoff formulation could be treated as a concave optimization problem. Finally, using Lagrangian method, an optimal power allocation strategy is provided.

\section{System ModeL}

We focus on a multiplexing-based system, which at the transmitter side, transmits different data streams through different subchannels and at the receiver side, recovers the parallel data streams separately [14]. To be specific, a wireless frequency-selective fading channel with a total bandwidth of $B$ is considered. In order to combat frequency selectivity, $N$ independent identically distributed (i.i.d.) flat-fading subchannels, each with a bandwidth of $B / N$, are employed.

Similar with the multi-carrier system model which is considered in [15], firstly, the upper-layer packets are divided into frames at the data-link layer. The frames are stored at the transmit buffer and split into bit streams at the physical layer. Then, based on the channel-state information (CSI) and QoS constraint, adaptive modulation and coding (AMC) and power policy are applied at the transmitter [14]. Then, the bit streams are read out of the buffer and transmitted through $N$ subcarriers.

Since each subcarrier is assumed to experiencing block fading, the channel gains of $N$ subcarriers are invariant within a fading-block's time duration $T_{\mathrm{f}}$, but independently varies from one block to anther. In addition, the length of each fading-block, $T_{\mathrm{f}}$, as an integer multiple of the symbol duration $T_{\mathrm{s}}$, is assumed to be less than the fading coherence time [14].

For the $n^{\text {th }}$ subcarrier at fading-block index $t$, the subcarrier power gain is denoted as $\left\{\gamma_{n}[t], n \in \mathcal{N}_{0}\right\}$, where $\mathcal{N}_{0}=$ $\{1,2, \ldots, N\}$ represents the set of all subcarriers. The joint probability density function (PDF) of the subchannel power gains, $\gamma[t]=\left[\begin{array}{llll}\gamma_{1}[t] & \gamma_{2}[t] & \ldots & \gamma_{N}[t]\end{array}\right]$, is given by $\rho(\gamma)$. Each subcarrier is also assumed to experience i.i.d. additive white Gaussian noise (AWGN) with power spectral density $\frac{\eta_{0}}{2}$.

Assuming Shannon capacity can be achieved, the total instantaneous service rate of the multi-carrier system at the $t^{\text {th }}$ fading-block is then given as

$R[t]=\frac{1}{N} \sum_{n=1}^{N} \log _{2}\left(1+P_{n}(\theta, \gamma[t]) \cdot \frac{\gamma_{n}[t]}{P_{\mathcal{L}} \eta_{0}\left(\frac{B}{N}\right)}\right)$

where $P_{\mathcal{L}}$ denotes the distance-based path-loss power and $P_{n}(\theta, \gamma[t]), n \in \mathcal{N}_{0}$, as a function of $\theta$ and $\gamma[t]$, are the transmission powers for the $n^{\text {th }}$ subcarrier. Since the service rate process $\left\{R[t], t=1,2, \ldots, T_{\mathrm{f}}\right\}$ is considered to be stationary and ergodic [14], the block index $t$ could be omitted for simplicity.

\section{A. Effective Capacity}

Considering a queue of infinite buffer size served by a channel of constant service rate, the probability of the queue length exceeding a threshold $x$ can be derived using the large deviation theory [16], which satisfies

$$
-\lim _{x \rightarrow \infty} \frac{\ln (\operatorname{Pr}\{Q(\infty) \geq x\})}{x}=\theta,
$$

where $\operatorname{Pr}\{a \geq b\}$ shows the probability of $a \geq b, Q(\infty)$ shows the steady-state queue length. Note that in (2), the parameter $\theta(\theta>0)$ indicates the exponential decay rate of the QoS violation probability. A smaller value of $\theta$ denotes a looser QoS requirement, while larger $\theta$ represents a more stringent delay constraint. Particularly, when $\theta \rightarrow 0$, there is no delay constraint, which means the system can tolerate unlimited delay.

Now, assuming that the Gartner-Ellis theorem [17, Pages 34-36] is satisfied, EC of a multi-carrier system with $N$ i.i.d subchannels can be expressed as [10]

$$
E_{\mathrm{c}}(\theta)=-\frac{1}{\theta T_{\mathrm{f}} B} \ln \left(\mathbb{E}\left[e^{-\theta B T_{\mathrm{f}} R[t]}\right]\right) \quad(\mathrm{b} / \mathrm{s} / \mathrm{Hz}),
$$

where $\mathbb{E}[\cdot]$ indicates the expectation operator. Substituting (1) into (3), EC can be further expressed as

$$
E_{\mathrm{c}}(\theta)=-\frac{1}{\alpha} \log _{2}\left(\mathbb{E}_{\gamma}\left[\prod_{n=1}^{N}\left(1+\frac{P_{n}(\theta, \gamma) \gamma_{n}}{P_{\mathcal{L}} \eta_{0}\left(\frac{B}{N}\right)}\right)^{-\frac{\alpha}{N}}\right]\right),
$$

where $\alpha \equiv \frac{\theta T_{\mathrm{f}} B}{\ln (2)}, \mathbb{E}_{\boldsymbol{\gamma}}[\cdot]$ indicates the expectation over the PDF of $\boldsymbol{\gamma}, \boldsymbol{P}(\theta, \boldsymbol{\gamma})=\left[\begin{array}{llll}P_{1}(\theta, \gamma) & P_{2}(\theta, \gamma) & \ldots & P_{N}(\theta, \gamma)\end{array}\right]$ denotes a $N \times 1$ vector of subcarrier power allocations.

Then, the delay-outage probability, which is defined as the probability that the delay exceeds a maximum delay-bound $D_{\max }$, can be estimated as [10]

$$
P_{\text {delay }}^{\text {out }}=\operatorname{Pr}\left\{\text { Delay } \geq D_{\max }\right\} \approx \varepsilon \mathrm{e}^{-\theta \mu D_{\max }},
$$

where $D_{\max }$ is in units of a symbol period $\left(T_{\mathrm{s}}=1 / B\right)$. For a given $\mu, \varepsilon=\operatorname{Pr}\{Q(t) \geq 0\}$ is the probability that the buffer is nonempty at time $t$ and can be approximated as the ratio of the constant arrival rate to the average service rate [16], i.e., $\varepsilon \simeq \frac{\mu}{\mathbb{E}[R[t]]}$. Hence, in order to meet a target delay-outage probability limit $P_{\text {delay, }}^{\text {out }}$ a source needs to limit its data rate to the maximum of $\mu$, where $\mu=E_{\mathrm{c}}(\theta)$, given in (3).

\section{B. Energy Efficiency}

We formulate the link-layer EE of a multi-carrier system as the ratio of EC to the sum of the circuit power, $P_{\mathrm{c}}$, and the 
transmission power scaled by the power amplifier efficiency $\epsilon$. Therefore, EE can be mathematically expressed as

$$
\operatorname{EE}(\theta)=\frac{-\frac{1}{\alpha} \log _{2}\left(\mathbb{E}_{\gamma}\left[\prod_{n=1}^{N}\left(1+\frac{N P_{n}(\theta, \gamma) \gamma_{n}}{K_{\ell}}\right)^{-\frac{\alpha}{N}}\right]\right)}{P_{\mathrm{c}}+\frac{1}{\epsilon} \cdot \mathbb{E}_{\gamma}\left[\sum_{n=1}^{N} P_{n}(\theta, \gamma)\right]},
$$

where $K_{\ell}=P_{\mathcal{L}} \eta_{0} B$ is the path loss factor, including both AWGN power and path loss power.

\section{Optimal Power Allocation}

In this section, for a multi-carrier link with $N$ i.i.d subcarriers, we propose an MOP approach to integrate the linklayer EE-EC tradeoff problem, and solve it using advanced lexicographic method and Lagrangian method.

Firstly, in order to jointly maximize the two conflicting objectives, EE and EC, an MOP could be formulated as

$$
\max \operatorname{EE}(\theta) \text { and } \max E_{\mathrm{c}}(\theta) \text {. }
$$

In order to solve the MOP, we convert it into an SOP using the advanced lexicographic method, which arranges the objective functions in order of their importance [18]. To be specific, EC will be maximized subject to a constraint on the required EE level. Therefore, in a multi-carrier system, the proposed EE-EC tradeoff formulation can be mathematically expressed as

$$
\begin{aligned}
& E_{\mathrm{c}}^{\mathrm{opt}}(\theta)= \\
& \max _{\substack{n \\
P_{n}^{\mathrm{r}}(\theta, \gamma) \geq 0 \\
n \in \mathcal{N}_{0}}}-\frac{1}{\alpha} \log _{2}\left(\mathbb{E}_{\boldsymbol{\gamma}}\left[\prod_{n=1}^{N}\left(1+N P_{n}^{\mathrm{r}}(\theta, \gamma) \gamma_{n}\right)^{-\frac{\alpha}{N}}\right]\right)
\end{aligned}
$$

s.t.

$$
\frac{-\frac{1}{\alpha} \log _{2}\left(\mathbb{E}_{\gamma}\left[\prod_{n=1}^{N}\left(1+N P_{n}^{\mathrm{r}}(\theta, \gamma) \gamma_{n}\right)^{-\frac{\alpha}{N}}\right]\right)}{K_{\ell}\left(P_{\mathrm{c}_{\mathrm{r}}}+\frac{1}{\epsilon} \cdot \mathbb{E}_{\gamma}\left[\sum_{n=1}^{N} P_{n}^{\mathrm{r}}(\theta, \gamma)\right]\right)} \geq \eta_{\min }(\theta),
$$

where $P_{n}^{\mathrm{r}}(\theta, \gamma)=\frac{P_{n}(\theta, \gamma)}{K_{\ell}}, P_{\mathrm{c}_{\mathrm{r}}}=\frac{P_{\mathrm{c}}}{K_{\ell}}$. Further, $\eta_{\min }(\theta)$ represents the required $\mathrm{EE}$ level and is defined by a certain ratio of the maximum achievable $\mathrm{EE}$ at the target delayoutage probability. Specifically, similar to [13], in order to flexibly control the tradeoff level, an adjustable indicator, EE-loss-rate $\left(\alpha_{\mathrm{EE}}\right)$, is introduced, which controls the level of the required EE limit $\eta_{\min }(\theta)$, according to

$$
\eta_{\min }(\theta)=\alpha_{\mathrm{EE}} \cdot \eta_{\max }(\theta),
$$

where $\eta_{\max }(\theta)$ represents the maximum achievable EE of the multi-carrier system and $0 \leq \alpha_{\mathrm{EE}} \leq 1$. In particular, when $\alpha_{\mathrm{EE}}=0$, the EE constraint limit $\eta_{\min }(\theta)=0$, which indicates that the tradeoff problem is simplified into an ECmaximization problem. In contrast, when $\alpha_{\mathrm{EE}}=1$, the EE constraint limit $\eta_{\min }(\theta)=\eta_{\max }(\theta)$, which results in an EEmaximization problem.

Setting $\eta_{\min }^{\prime}(\theta)=\eta_{\min }(\theta) K_{\ell}$, then, $K_{\ell}$ in (7a)( $7 \mathrm{~b})$ could be canceled to scale the system performance with respect to the path loss factor. Further, the multicarrier transmit power is referred to as $\boldsymbol{P}^{\mathrm{r}}(\theta, \gamma)=$
$\left[\begin{array}{llll}P_{1}^{\mathrm{r}}(\theta, \gamma) & P_{2}^{\mathrm{r}}(\theta, \gamma) & \ldots & P_{N}^{\mathrm{r}}(\theta, \gamma)\end{array}\right]$ and the EE-EC tradeoff formulation can be re-formulated as

$$
\begin{aligned}
& E_{\mathrm{c}}^{\mathrm{opt}}(\theta)= \\
& \max _{\substack{P_{n}^{\mathrm{r}}(\theta, \gamma) \geq 0 \\
n \in \mathcal{N}_{0}}}-\frac{1}{\alpha} \log _{2}\left(\mathbb{E}_{\boldsymbol{\gamma}}\left[\prod_{n=1}^{N}\left(1+N P_{n}^{\mathrm{r}}(\theta, \gamma) \gamma_{n}\right)^{-\frac{\alpha}{N}}\right]\right) \\
& \text { s.t. } \frac{-\frac{1}{\alpha} \log _{2}\left(\mathbb{E}_{\boldsymbol{\gamma}}\left[\prod_{n=1}^{N}\left(1+N P_{n}^{\mathrm{r}}(\theta, \gamma) \gamma_{n}\right)^{-\frac{\alpha}{N}}\right]\right)}{P_{\mathrm{c}_{\mathrm{r}}}+\frac{1}{\epsilon} \cdot \mathbb{E}_{\boldsymbol{\gamma}}\left[\sum_{n=1}^{N} P_{n}^{\mathrm{r}}(\theta, \gamma)\right]} \geq \eta_{\min }^{\prime}(\theta) .
\end{aligned}
$$

The objective function (9a) has been proved to be concave in $\boldsymbol{P}^{\mathrm{r}}(\theta, \gamma)$ in [15]. Further, EE, as a ratio of a concave over a non-negative affine function in $\boldsymbol{P}^{\mathrm{r}}(\theta, \gamma)$, is a quasi-concave function of the subcarrier power allocations [15]. Therefore, its upper contour set defined by (9b) is convex [19]. Hence (9a)(9b) is a concave optimization problem and the Karush-KuhnTucker (KKT) conditions are both sufficient and necessary for the global optimum value. The constraint $(9 b)$ can be further transformed to

$$
\begin{aligned}
& -\frac{1}{\alpha} \log _{2}\left(\mathbb{E}_{\boldsymbol{\gamma}}\left[\prod_{n=1}^{N}\left(1+N P_{n}^{\mathrm{r}}(\theta, \gamma) \gamma_{n}\right)^{-\frac{\alpha}{N}}\right]\right) \\
& -\eta_{\min }^{\prime}(\theta)\left(P_{\mathrm{c}_{\mathrm{r}}}+\frac{1}{\epsilon} \cdot \mathbb{E}_{\boldsymbol{\gamma}}\left[\sum_{n=1}^{N} P_{n}^{\mathrm{r}}(\theta, \gamma)\right]\right) \geq 0 .
\end{aligned}
$$

Considering $\lambda \in R$ is the Lagrange multiplier associated to (10), the Lagrangian is

$$
\begin{aligned}
& \mathcal{L}\left(\boldsymbol{P}^{\mathrm{r}}(\theta, \gamma), \lambda\right) \\
& =-\frac{1}{\alpha} \log _{2}\left(\mathbb{E}_{\boldsymbol{\gamma}}\left[\prod_{n=1}^{N}\left(1+N P_{n}^{\mathrm{r}}(\theta, \gamma) \gamma_{n}\right)^{-\frac{\alpha}{N}}\right]\right) \\
& +\lambda\left(-\frac{1}{\alpha} \log _{2}\left(\mathbb{E}_{\boldsymbol{\gamma}}\left[\prod_{n=1}^{N}\left(1+N P_{n}^{\mathrm{r}}(\theta, \gamma) \gamma_{n}\right)^{-\frac{\alpha}{N}}\right]\right)\right. \\
& \left.-\eta_{\min }^{\prime}(\theta)\left(P_{\mathrm{c}_{\mathrm{r}}}+\frac{1}{\epsilon} \cdot \mathbb{E}_{\boldsymbol{\gamma}}\left[\sum_{n=1}^{N} P_{n}^{\mathrm{r}}(\theta, \gamma)\right]\right)\right) \\
& -\sum_{n=1}^{N} \mu_{n} P_{n}^{\mathrm{r}}(\theta, \gamma) .
\end{aligned}
$$

At the optimal power allocation, we have

$$
\frac{\partial \mathcal{L}\left(\boldsymbol{P}^{\mathrm{r}}(\theta, \boldsymbol{\gamma}), \lambda\right)}{\partial \boldsymbol{P}^{\mathrm{r}}(\theta, \gamma)}=0
$$

Because of the complementary slackness [19], if $P_{n}^{\mathrm{r}}(\theta, \gamma)>$ $0, \forall n \in \mathcal{N}_{0}$, then $\mu_{n}=0, \forall n \in \mathcal{N}_{0}$. On the other hand, if $P_{n}^{r}(\theta, \gamma)=0, \exists n \in \mathcal{N}_{0}$, then $\mu_{n} \neq 0$. Thus, the following two cases need to be considered to find the optimal power allocations. Note that $P_{n}^{\mathrm{r}}(\theta, \gamma)$ will be denoted as $P_{n}^{\mathrm{r}}$ for simplicity in the following analysis.

1) Case 1: $P_{n}^{r}>0, \forall n \in \mathcal{N}_{0}$ : In this situation, all $N$ subcarriers are allocated non-zero transmission power. Therefore, based on the complementary slackness, $\left\{\mu_{n}\right\}_{n=1}^{N}=0$. Then, 
the KKT condition (12) can be simplified as

$$
\left(1+N P_{n}^{\mathrm{r}} \gamma_{n}\right)^{-\frac{\alpha}{N}-1} \prod_{i \neq n}\left(1+N P_{i}^{\mathrm{r}} \gamma_{i}\right)^{-\frac{\alpha}{N}}=\frac{\beta}{\gamma_{n}} \forall n \in \mathcal{N}_{0}
$$

where $\beta=\frac{\lambda \eta_{\min }^{\prime}(\theta)}{\epsilon(\lambda+1) \log _{2} \mathrm{e}} \mathbb{E}_{\boldsymbol{\gamma}}\left[\prod_{n=1}^{N}\left(1+N P_{n}^{\mathrm{r}} \gamma_{n}\right)^{-\frac{\alpha}{N}}\right]$. By multiplying the right and left-hand sides of the $N$ equations in (13), the optimal power allocation strategy can be obtained as

$$
P_{n}^{\mathrm{r}}=\frac{1}{N}\left[\frac{1}{\beta^{\frac{1}{\alpha+1}} \prod_{i \in \mathcal{N}_{0}} \gamma_{i}^{\frac{\alpha}{(\alpha+1) N}}}-\frac{1}{\gamma_{n}}\right], \quad n \in \mathcal{N}_{0} .
$$

We note that $\beta$ acts as a cut-off threshold below which no power is allocated for transmission. The derived power allocation strategy (14) is optimal only when all subcarriers are assigned with positive powers. If there are one or more subcarriers which are allocated non-positive powers, then a second case needs to be taken into consideration.

2) Case 2: $P_{m}^{r}=0, \exists m \in \mathcal{N}_{0}$ : If there exists $P_{m}^{\mathrm{r}} \leq 0$, then the set of subcarriers, which only positive powers should be assigned, needs to be found.

Firstly, we define $\mathcal{N}_{1}$ as

$$
\mathcal{N}_{1}=\left\{n \in \mathcal{N}_{0} \mid \frac{1}{N}\left[\frac{1}{\beta^{\frac{1}{\alpha+1}} \prod_{i \in \mathcal{N}_{0}} \gamma_{i}^{\frac{\alpha}{(\alpha+1) N}}}-\frac{1}{\gamma_{n}}\right] \geq 0\right\} \text {. }
$$

According to Lemma 1 in [14], the total power must be assigned to the subcarriers which belong to $\mathcal{N}_{1}$, while the subcarriers $n \notin \mathcal{N}_{1}$ should not be allocated any power. Therefore, a new optimization problem could be expressed as

$$
\begin{aligned}
& E_{\mathrm{c}}^{\mathrm{opt}}(\theta)= \\
& \max _{\substack{n \\
P_{n}^{\mathrm{r}}(\theta, \gamma) \geq 0 \\
n \in \mathcal{N}_{1}}}-\frac{1}{\alpha} \log _{2}\left(\mathbb{E}_{\boldsymbol{\gamma}}\left[\prod_{n=1}^{N_{1}}\left(1+N P_{n}^{\mathrm{r}}(\theta, \gamma) \gamma_{n}\right)^{-\frac{\alpha}{N}}\right]\right)
\end{aligned}
$$

$$
\begin{aligned}
& \text { s.t. } \\
& \frac{-\frac{1}{\alpha} \log _{2}\left(\mathbb{E}_{\boldsymbol{\gamma}}\left[\prod_{n=1}^{N_{1}}\left(1+N P_{n}^{\mathrm{r}}(\theta, \gamma) \gamma_{n}\right)^{-\frac{\alpha}{N}}\right]\right)}{P_{\mathrm{c}_{\mathrm{r}}}+\frac{1}{\epsilon} \cdot \mathbb{E}_{\boldsymbol{\gamma}}\left[\sum_{n=1}^{N_{1}} P_{n}^{\mathrm{r}}(\theta, \gamma)\right]} \geq \eta_{\min }^{\prime}(\theta),
\end{aligned}
$$

where $N_{1}=\left|\mathcal{N}_{1}\right|$ represents the cardinality of $\mathcal{N}_{1}$. Therefore, if $P_{n}^{\mathrm{r}}>0, \forall n \in \mathcal{N}_{1}$, then, the optimization problem can be solved exactly like Case 1 . Otherwise, if there are subcarriers $n \in \mathcal{N}_{1}$ having $P_{n}^{\mathrm{r}}=0$, then $\mathcal{N}_{1}$ must be further partitioned by recursively repeating the above process until a set $\mathcal{N}^{*}$ can be found, in which all subcarriers are allocated positive powers [15] (i.e., if $\mathcal{N}_{k}=\mathcal{N}_{k-1}=\left\{n \in \mathcal{N}_{k} \mid P_{n}^{\mathrm{r}}>0\right\}$, then $\mathcal{N}^{*}=$ $\left.\mathcal{N}_{k}\right)$.

After obtaining $\mathcal{N}^{*}$, the optimal power allocations are computed as

$P_{n}^{\mathrm{r}}= \begin{cases}\frac{1}{N}\left[\frac{1}{\beta^{\frac{N}{N+\alpha N^{*}}} \prod_{i \in \mathcal{N}^{*}} \gamma_{i}^{\overline{N+\alpha N^{*}}}}-\frac{1}{\gamma_{n}}\right], & n \in \mathcal{N}^{*} \\ 0, & \text { otherwise }\end{cases}$ where $N^{*}=\left|\mathcal{N}^{*}\right|$.

The optimal value for $\beta$, referred to as $\beta^{*}$, is found when EE constraint is satisfied with equality, yielding

$$
\begin{aligned}
& -\frac{1}{\alpha} \log _{2}\left(\mathbb{E}_{\gamma}\left[\prod_{n=1}^{N}\left(1+N P_{n}^{\mathrm{r}}(\theta, \gamma) \gamma_{n}\right)^{-\frac{\alpha}{N}}\right]\right) \\
& -\eta_{\min }^{\prime}(\theta)\left(P_{\mathrm{c}_{\mathrm{r}}}+\frac{1}{\epsilon} \cdot \mathbb{E}_{\gamma}\left[\sum_{n=1}^{N} P_{n}^{\mathrm{r}}(\theta, \gamma)\right]\right)=0 .
\end{aligned}
$$

After obtaining $\beta^{*}$, the average input power level could be found by

$$
\overline{P^{*}}=K_{\ell} \times\left.\mathbb{E}_{\gamma}\left[\sum_{n=1}^{N}\left(P_{n}^{\mathrm{r}}\right)\right]\right|_{\beta=\beta^{*}} .
$$

Then, the tradeoff problem is transformed into an ECmaximization problem, with a power constraint limit $\bar{P}^{*}$, which yields

$$
\begin{aligned}
& E_{\mathrm{c}}^{\mathrm{opt}}(\theta)= \\
& \max _{\substack{P_{n}^{\mathrm{r}}(\theta, \gamma) \geq 0 \\
n \in \mathcal{N}_{0}}}-\frac{1}{\alpha} \log _{2}\left(\mathbb{E}_{\gamma}\left[\prod_{n=1}^{N}\left(1+N P_{n}^{\mathrm{r}}(\theta, \gamma) \gamma_{n}\right)^{-\frac{\alpha}{N}}\right]\right) \\
& \text { s.t. } \quad \mathbb{E}_{\gamma}\left[\sum_{n=1}^{N} P_{n}^{\mathrm{r}}(\theta, \gamma)\right] \leq \frac{\overline{P^{*}}}{K_{\ell}}
\end{aligned}
$$

\section{NumERICAL RESUlts}

We numerically evaluate the performance of the multicarrier EE-EC tradeoff formulation over a Rayleigh frequencyselective fading channel, subject to delay-outage probability and EE constraints. We firstly assume that the $N$ i.i.d. subcarriers' power gains, i.e., $\gamma_{1}, \gamma_{2}, \ldots, \gamma_{n}$, are exponentially distributed with unit mean. In the following simulations, we assume that $B \cdot T_{\mathrm{f}}=200$, the circuit-to-noise power ratio $P_{\mathrm{c}_{\mathrm{r}}}=0.1 \mathrm{~W}$.

We start by plotting EC (on the left-hand-side (LHS) yAxis) and EE (on the right hand side (RHS) y-Axis) of the considered multi-carrier system versus EE-loss-rate $\left(\alpha_{\mathrm{EE}}\right)$, for various numbers of subcarriers, $N$ in Fig. 1. This figure indicates that with fixed $N$, when $\alpha_{\mathrm{EE}}$ increases, EE increases and EC decreases. This is due to the fact that larger $\alpha_{\mathrm{EE}}$ indicates more stringent EE constraint, which leads to a larger EE. Since the tradeoff system operates in the conflicting region of EE and EC, the EE-increases result from EC-reductions. At extreme cases, as shown in Section III, the maximum EC and the maximum EE are obtained at $\alpha_{\mathrm{EE}}=0$ and $\alpha_{\mathrm{EE}}=1$, respectively. Moreover, with fixed $\alpha_{\mathrm{EE}}$, as the number of subcarriers increases, both EC and EE increase.

We then plot the results for $\mathrm{EE}$ and $\mathrm{EC}$ of the multi-carrier system versus EE-loss-rate $\left(\alpha_{\mathrm{EE}}\right)$, for two different values of delay QoS exponent $\theta$ and various values of $N$, in Fig. 2 . With fixed $N$, when the delay QoS exponent $\theta$ decreases, both EE and EC increase. Especially, when $N$ is small, the increases of $\mathrm{EE}$ and $\mathrm{EC}$, as a result of the decrease in $\theta$, are significant. While when $N$ is larger, e.g., $N=8$, the increases of EE and $\mathrm{EC}$ are minor.

Link-layer $\mathrm{EE}$ versus the number of subcarriers, $N$, for various values of $\theta$ is plotted in Fig. 3. This figure shows that, for a determined tradeoff level with fixed $\alpha_{\mathrm{EE}}$, when the 


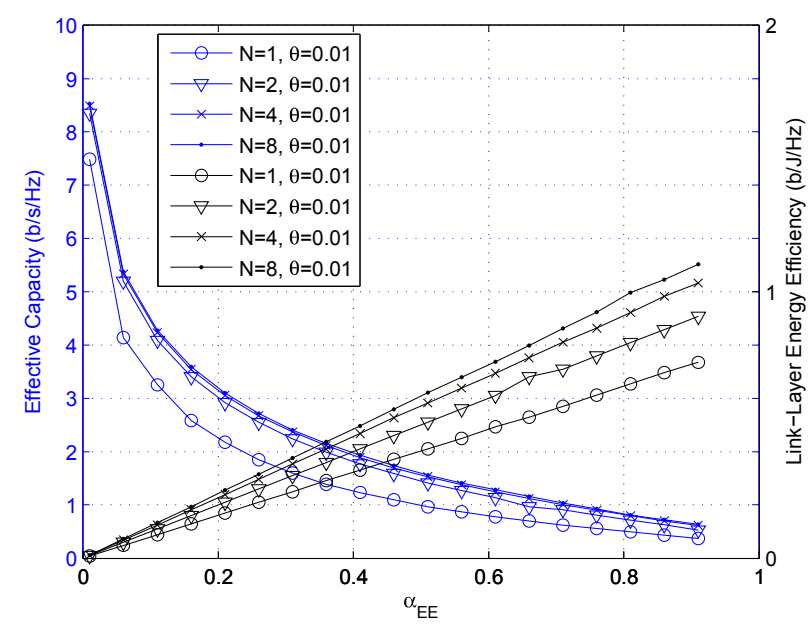

Fig. 1: Effective capacity and energy efficiency versus $\alpha_{\mathrm{EE}}$, for various values of $N$.

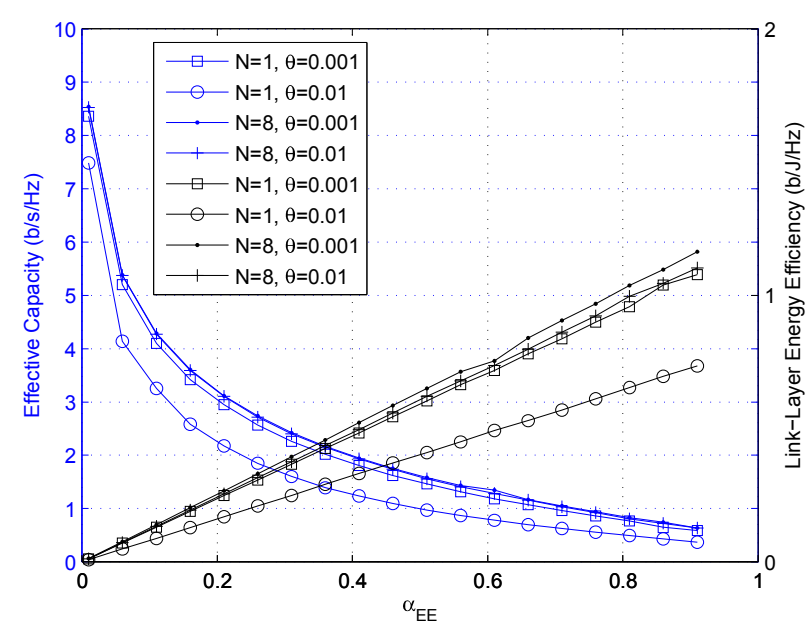

Fig. 2: Effective capacity and energy efficiency versus $\alpha_{\mathrm{EE}}$, for various values of $\theta$ and $N$.

number of subcarriers is low, EE will be easily influenced by the delay QoS exponent. In other words, it turns out that the system with more subcarriers is more robust with changes in $\theta$. In addition, in Fig. 3, with fixed $N$, when $\alpha_{\mathrm{EE}}=0.8$, EE in $\theta=0.001$ is always larger than $\theta=0.1$. This indicates that, in comparison with stringent Qos requirement system, EE in loose delay-constrained system could be sacrificed more to achieve an larger increase in EC.

Finally, the delay-outage probability, $P_{\text {delay, }}^{\text {out }}$ versus delay QoS exponent $\theta$, for various values of $\alpha_{\mathrm{EE}}$ with a maximum tolerable delay threshold $D_{\max }=200$, is plotted in Fig. 4 . The figure reveals that for loose delay-constrained systems, e.g., $\theta=10^{-4}$, the achievable $P_{\text {delay }}^{\text {out }}$ will stay the same with different $\alpha_{\mathrm{EE}}$. When the delay requirement is more stringent, e.g., $\theta=10^{-2}$, smaller $\alpha_{\mathrm{EE}}$ will provide smaller delay-outage probability. This happens because smaller $\alpha_{\mathrm{EE}}$ results in more sacrifices of $\mathrm{EE}$ from its maximum values, i.e., $\eta_{\max }(\theta)$, and in turn, more increases in EC. Therefore, the probability that the data length exceeds the buffer size decreases, and in turn, the delay-outage probability decreases.

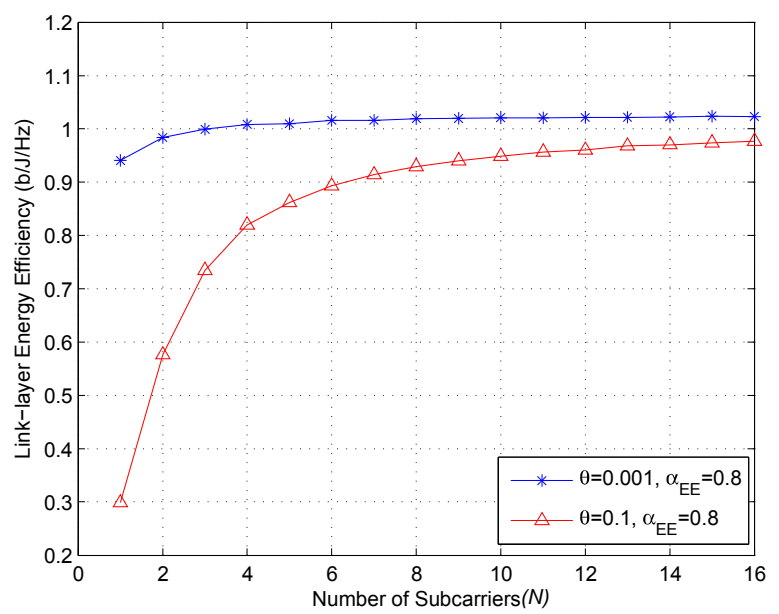

Fig. 3: Link-layer energy efficiency versus $N$, for various values of $\theta$.

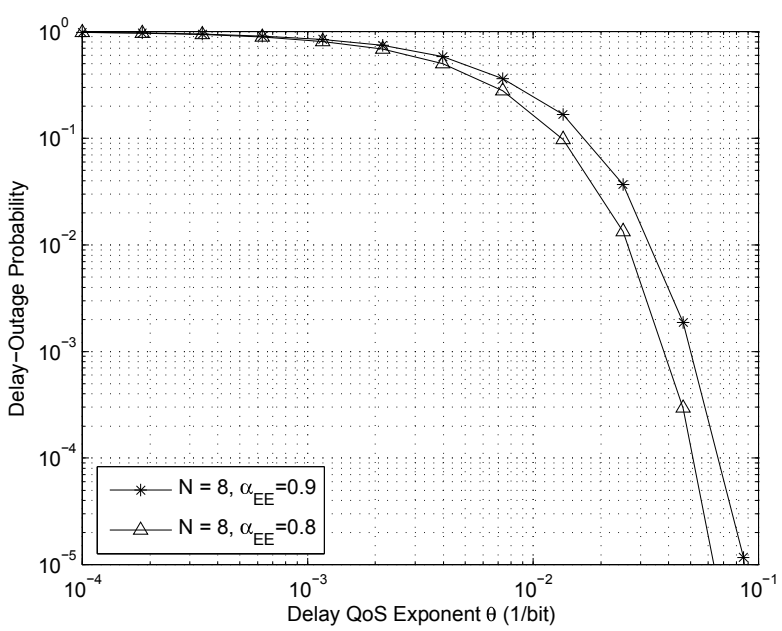

Fig. 4: Delay-outage probability versus delay QoS exponent $\theta$, for various values of $\alpha_{\mathrm{EE}}$.

\section{CONCLusions}

For a multi-carrier channel with $N$ i.i.d flat-fading subcarriers, we formulated the link-layer EE-EC tradeoff problem using an MOP approach, and solved it using advanced lexicographic method and Lagrangian method. To be specific, the system EC was maximized subject to an EE constraint. By introducing the adjustable performance indicator, EE-loss-rate $\left(\alpha_{\mathrm{EE}}\right)$, into the EE constraint, the constraint limit and the tradeoff level were flexibly controlled. We then proved that the tradeoff formulation is a concave maximization problem and could be solved using Lagrangian method. Finally, the optimal power allocation strategy was provided, carrying a similar structure with the power allocation technique in EE-maximization problem for a frequency-selective fading channel, but with a different cut-off threshold.

\section{REFERENCES}

[1] M. H. Alsharif, R. Nordin, and M. Ismail, "Survey of green radio communications networks: Techniques and recent advances," Journal of Computer Networks and Communications, Nov. 2013. 
[2] C. Gunaratne, K. Christensen, B. Nordman, and S. Suen, "Reducing the energy consumption of ethernet with adaptive link rate (ALR)," IEEE Trans. on Computers, vol. 57, no. 4, pp. 448-461, Apr. 2008.

[3] Smart2020, "Enabling the low-carbon economy in the information age," The Climate Group, London, U.K., Tech. Rep., 2008.

[4] W. D. Nordhaus, "To slow or not to slow: The economics of the greenhouse effect," The Economic Journal, vol. 101, no. 407, pp. 920937, Jul. 1991.

[5] K. Lahiri, A. Raghunathan, S. Dey, and D. Panigrahi, "Battery-driven system design: A new frontier in low power design," in IEEE Int. Conf. on VISI Design, Bangalore, India, Jan. 2002, pp. 261-267.

[6] C. Xiong, G. Y. Li, S. Zhang, Y. Chen, and S. Xu, "Energy- and spectral-efficiency tradeoff in downlink OFDMA networks," IEEE Trans. Wireless Commun., vol. 10, no. 11, pp. 3874-3886, Nov. 2011.

[7] X. Chen and S. Ouyang, "Energy- and spectral-efficiency trade-off in OFDMA-based cooperative cognitive radio networks," International Journal of Distributed Sensor Networks, Feb. 2014.

[8] J. Tang, D. K. C. So, E. Alsusa, and K. A. Hamdi, "Resource efficiency: A new paradigm on energy efficiency and spectral efficiency tradeoff," IEEE Trans. on Wireless Communications, vol. 13, no. 8, pp. 1536-1276, Aug. 2014.

[9] O. Amin, E. Bedeer, M. H. Ahmed, and O. A. Dobre, "Energy efficiency and spectral efficiency trade-off for OFDM systems with imperfect channel estimation," in IEEE Int. Conf. Commun. (ICC 2014), Sydney, Australia, Jun. 2014, pp. 3553-3558.

[10] D. Wu and R. Negi, "Effective capacity: A wireless link model for support of quality-of-service," IEEE Trans. Wireless Commun., vol. 2, no. 4, pp. 630-643, Jul. 2003.

[11] G. Li, Z. Xu, C. Xiong, C. Yang, S. Zhang, Y. Chen, and S. Xu, "Energyefficient wireless communications: tutorial, survey, and open issues," IEEE Wireless Commun., vol. 18, no. 6, pp. 28-35, Dec. 2011.

[12] X. Chen, R. Q. Hu, G. Wu, and Q. C. Li, "Tradeoff between energy efficiency and spectral efficiency in a delay constrained wireless system," Wireless Communications and Mobile Computing, Mar. 2014.

[13] L. Musavian and Q. Ni, "Delay-QoS-driven spectrum and energy efficiency tradeoff," in IEEE Int. Conf. Commun. (ICC 2014), Sydney, Australia, Jun. 2014.

[14] J. Tang and X. Zhang, "Quality-of-service driven power and rate adaptation for multichannel communications over wireless links," IEEE Trans. Wireless Commun., vol. 6, no. 12, pp. 1536-1276, Dec. 2007.

[15] A. Helmy, L. Musavian, and T. Le-Ngoc, "Energy-efficient power adaptation over a frequency-selective fading channel with delay and power constraints," IEEE Trans. Wireless Commun., vol. 12, no. 9, pp. 4529-4541, Sep. 2013.

[16] C. S. Chang, "Stability, queue length, and delay of deterministic and stochastic queueing networks," IEEE Trans. Automatic Control, vol. 39, no. 5, pp. 913-931, May 1994.

[17] J. A. Bucklew, Introduction to Rare Event Simulation. Springer-Verlag New York Inc., 2004.

[18] J. S. A. R. T. Marler, "Survey of multi-objective optimization methods for engineering," Structural and Multidisciplinary Optimization, vol. 26, no. 6, pp. 369-395, Apr. 2004.

[19] S. Boyd and L. Vandenberghe, Convex Optimization. Cambridge University Press, 2004. 